\title{
Associação entre risco de disfagia e risco nutricional em idosos internados em hospital universitário de Brasília
}

\author{
Association between risk of dysphagia and \\ nutritional risk in elderly inpatients at a \\ Univesity Hospital of Brasília, Brazil
}

Juliana Rolim Vieira MACIEL ${ }^{1}$

Carlos Jorge Rocha OLIVEIRA ${ }^{2,3,4}$

Cristiane de Melo Pantaleão TADA ${ }^{5}$

\section{RE S U M O}

\section{Objetivos}

Investigar a associação entre o grau de risco de disfagia e o risco nutricional dos pacientes idosos internados na clínica médica do Hospital Universitário de Brasília.

\section{Métodos}

Estudo transversal, analítico, original, observacional, não-controlado, com amostra de conveniência e estratificada. Foram aplicados a Mini Avaliação Nutricional e o questionário de risco de disfagia em 49 pacientes idosos nas primeiras 48 horas após admissão na clínica médica, em setembro de 2006.

\section{Resultados}

A freqüência de idosos foi maior na faixa etária entre 60-74 anos 11 meses (78\%), com média de idade de 69 anos, sendo $41 \%$ mulheres e $59 \%$ homens. A proporção de risco de disfagia dos pacientes idosos foi de $69 \%$ e do estado nutricional inadequado de $71 \%$, valor elevado principalmente entre as mulheres. Ambos os sexos se encontram em risco nutricional, masculino (20,5, Desvio-padrão - DP=5) e feminino (19, DP=6). O estudo mostrou que as mulheres apresentam uma tendência maior para risco de disfagia, porém os homens apresentam um quadro de maior gravidade (risco moderado). Foi encontrada uma correlação negativa moderada entre os valores de risco de disfagia e seus respectivos scores da Mini Avaliação Nutricional.

\footnotetext{
1 Hospital Universitário de Brasília, Divisão de Nutrição. SGAN 604, HUB, 70840-060, Brasília, DF, Brasil. Correspondência para/Correspondence to: J.R.V. MACIEL. E-mail: <juliana.rolim@gmail.com>.

2 Universidade Anhembi Morumbi de São Paulo. São Paulo, SP, Brasil

${ }^{3}$ Universidade Santamarense de São Paulo. São Paulo, SP, Brasil

${ }^{4}$ Instituto de Pesquisa e Capacitação e Especialização. São Paulo, SP, Brasil.

5 GANEP-Nutrição Humana, Especialização em Nutrição Clínica. São Paulo, SP, Brasil.
} 


\section{Conclusão}

Foi verificado neste estudo que os idosos apresentam dois agravantes de sua qualidade de vida: estado nutricional e disfagia, que podem ser agudizados na internação hospitalar.

Termos de indexação: Avaliação nutricional. Desnutrição. Idoso. Transtornos de deglutição.

\section{A B S T R A C T}

\section{Objectives}

This study aimed to verify the association between the degree of risk of dysphagia and nutritional risk of elderly inpatients at the medical clinic of Brasilia's University Hospital.

\section{Methods}

This is a cross-sectional, analytic, original, observational, uncontrolled study with convenience and stratified sample. The Mini Nutritional Assessment and a questionnaire to assess risk of dysphagia were administered to 49 elderly patients within the first 48 hours after admission to the medical clinic, in September 2006.

\section{Results}

Most of the elderly inpatients aged from 60 to 74 years and 11 months (78\%) with a mean age of 69 years; $41 \%$ were females and $59 \%$ were males. Sixty-nine percent of the elderly inpatients were at risk of dysphagia and $71 \%$ presented inadequate nutritional status, especially women. Both males $(20.5$, standard deviation = 5) and females (19, standard deviation =6) were at nutritional risk. The study showed that women are more likely to develop dysphagia yet men presented a higher degree of risk (moderate risk). There is a moderate negative correlation between the values obtained for the risk of dysphagia and their respective Mini Nutritional Assessment scores.

\section{Conclusion}

This study verified that two factors aggravate the quality of life of the elderly: their nutritional status and dysphagia. Both can worsen dramatically during hospitalization.

Indexing terms: Nutrition assessment. Malnutrition. Aged. Deglutition disorders.

\section{N T R O D U Ç Ã O}

O número de idosos vem apresentando um rápido crescimento em relação à população mundial, principalmente nos países em desenvolvimento, como é o caso do Brasil'1.

O último censo demográfico brasileiro do Instituto Brasileiro de Geografia e Estatística (IBGE) de 2000 aponta que, em 2006, havia 15 milhões de idosos (8,60\% da população brasileira). No Distrito Federal essa distribuição, corresponde a $5,07 \%$ da população geral, sendo $4,74 \%$ de homens e $5,37 \%$ de mulheres ${ }^{2}$.

O estudo do Inquérito Brasileiro de Avaliação Nutricional Hospitalar (IBRANUTRI) ${ }^{3}$ mostrou que a prevalência média da população brasileira de pacientes internados desnutridos é de $48,1 \%$, sendo que $31,8 \%$ dos pacientes já se encontram desnutridos nas primeiras 48 horas de internação, aumentando à medida em que o período de internação se prolonga.

Outros estudos sobre a desnutrição no ambiente hospitalar mostram que essa prevalência tem aumentado, variando entre 19 e $80 \%$ em pacientes clínicos e cirúrgicos, com índice maior entre idosos ${ }^{4-7}$. A variabilidade em torno da prevalência de desnutrição de pacientes internados é entre $2 \%$ e $82 \%{ }^{8,9}$ e essas diferenças são decorrentes da heterogeneidade da população envolvida nos diversos estudos ${ }^{10,11}$.

A avaliação nutricional deve ser aplicada a todos os pacientes internados, dentro de um prazo de 48 horas a partir da data de admissão na unidade hospitalar específica ${ }^{8}$.

Um dos métodos validado para tanto se destaca a Mini Avaliação Nutricional (MAN) ${ }^{12}$, que é um método prático, não invasivo, de simples 
mensurações e de questões rápidas, aplicadas em cerca de 10 minutos $^{12,13}$. A MAN foi utilizada com sucesso em acompanhamento da evolução clínica e da intervenção nutricional, de programas nutricionais educacionais para idosos. A detecção precoce de desnutrição é a chave da avaliação geriátrica $^{5,14-16}$.

Vários estudos comparativos entre a MAN e a Avaliaçao Nutricional Subjetiva Global (ANSG) em idosos foram realizados e verificaram que a MAN foi melhor preditor do estado de saúde e identificou necessidade de suporte nutricional antes da ANSG ${ }^{16-18}$.

Assim, a MAN é ferramenta mais apropriada nesses pacientes idosos do que a ANSG para identificar desnutrição severa. A MAN possui grande potencial em triar, avaliar e monitorar o risco nutricional, detectar a necessidade de medidas nutricionais preventivas, como o suporte nutricional precoce, e verificar o impacto da intervenção nutricional com melhora nos parâmetros nutricionais. Desse modo, pode ser utilizada facilmente na admissão hospitalar 13,17,18 .

Um fator identificado que interfere no estado nutricional do paciente internado é a disfagia, um distúrbio da deglutição, que é um processo fisiológico complexo, agravado pelo envelhecimento ${ }^{19}$.

A disfagia pode resultar de uma anormalidade anatômica ou funcional (neuromuscular) em qualquer estrutura e fase do processo de deglutição, como oral, faríngea ou esofagiana. Pode ocorrer em qualquer idade ${ }^{19}$.

Nos pacientes idosos, a prevalência exata de disfagia é desconhecida; freqüentemente aparece em cerca de $50 \%$ dos pacientes idosos internados. Isso decorre do processo de envelhecimento, por conta da diminuição ou dificuldade no processo de mastigação, por xerostomia, por desordens neurológicas (acidente vascular cerebral, doença de Parkinson, Mal de Alzheimer e câncer de cabeça e pescoço) e por desordens musculares e da anatomia orofaríngea ${ }^{20-22}$.
Assim, a disfagia contribui para aumentar a morb-mortalidade desses pacientes, com alterações na qualidade de vida, podendo provocar desidratação, desnutrição, asfixia, congestão pulmonar e infecções recorrentes do trato respiratório ${ }^{21}$.

O tratamento da disfagia em idosos é similar ao de adultos, porém terapias mais invasivas, como as cirurgias, podem não ser possíveis na maioria dos idosos, sendo os medicamentos menos agressivos e a terapia por endoscopia mais recomendados nesses casos ${ }^{23}$.

Essa faixa etária representa um fator de risco nutricional com possível agravamento na presença de disfagia, sendo recomendadas avaliação, elaboração e definição de rotinas e condutas destinadas à melhoria desse quadro.

O objetivo do estudo foi identificar a associação entre o grau de risco de disfagia e o risco nutricional dos pacientes idosos internados na clínica médica do Hospital Universitário de Brasília (HUB).

\section{MÉ TODOS}

O estudo foi do tipo transversal, analítico, observacional, não-controlado. Foi realizado com pacientes idosos admitidos na clínica médica no Hospital Universitário de Brasília, durante o mês de setembro de 2006.

Foi utilizada uma amostra de conveniência, estratificada, composta de 49 pacientes idosos, de ambos os sexos. Foram considerados como critérios de exclusão os pacientes acamados, sem mobilização, com algum membro amputado, em uso de próteses ortopédicas, com edema e com ascite.

Os pacientes foram recrutados no momento da admissão na clínica médica, com aplicação dos instrumentos de coleta de dados nas primeiras 48 horas após a internação.

A pesquisa foi aprovada pelo Comitê de Ética da Faculdade de Ciências da Saúde/Univer- 
sidade de Brasília, protocolo $n^{\circ}$ 0772006, e os pacientes autorizaram a participação por meio do Termo de Consentimento Livre e Esclarecido.

A avaliação nutricional do paciente iniciouse com a coleta de peso e estatura, conforme técnicas de mensuração descritas por Waitzberg \& Ferrini ${ }^{24}$.

O peso foi medido em balança digital Plenna ${ }^{\circledR}$, com escala de $100 \mathrm{~g}$ e capacidade de $150 \mathrm{~kg}$, com os pacientes descalços e em uso de roupa padrão hospitalar e sem nenhum adereço que interferisse no valor de aferição.

A estatura utilizada foi a estimada, por ser um método mais fidedigno nesse grupo populacional, sendo aferida a altura do joelho, utilizando fita métrica inextensível, conforme técnicas descritas por Chumlea et al..$^{25}$.

As posições utilizadas para essa aferição foram reclinada ou sentada. Na posição reclinada o paciente se encontra em decúbito dorsal, com o joelho curvado a um ângulo de $90^{\circ}$ e faz-se a medida do calcanhar à superfície anterior da coxa, próximo à patela. $\mathrm{O}$ estudo de Chumlea et al..$^{25}$ aponta que não há diferença estatística na mensuração entre essas duas posições; porém a medida em posição reclinada é mais acurada.

Após aferição, os dados eram introduzidos nas fórmulas abaixo de estimativa de estatura para idosos, definidas por Chumlea et al. ${ }^{25}$, sendo:

Homens: estatura $(\mathrm{cm})=[2,02 \times$ altura do joelho $(\mathrm{cm})]-[0,04 \mathrm{x}$ idade (anos) $]+64,19$

Mulheres: estatura $(\mathrm{cm})=[1,83 \times$ altura do joelho $(\mathrm{cm})]-[0,24 \mathrm{x}$ idade (anos) $]+84,88$

Em estudo de Sampaio et al. ${ }^{26}$, realizado para comparação das diferentes fórmulas de estimativa de estatura com as medidas obtidas diretamente em pacientes hospitalizados, verificou-se que, para os idosos, essas medidas se mostraram bem correlacionadas.

Após a coleta das medidas antropométricas, foram realizadas Mini Avaliação Nutricional ${ }^{12}$ e a avaliação do grau de disfagia com a aplicação de questionário específico.
A proporção de desnutrição foi determinada conforme a classificação da MAN. Esta classifica os indivíduos, segundo Guigoz et al. ${ }^{27}$, em estado nutricional adequado ( $\geq 24$ pontos), risco de desnutrição, entre 17 e 23,5 pontos; e desnutrição, com menos de 17 pontos. O questionário de disfagia, adaptado de Souza et al. ${ }^{28}$, classifica a disfagia em risco mínimo (0 a 1 ponto), risco leve (2 a 9 pontos), risco moderado (10 a 17 pontos) e risco grave (18 a 25 pontos).

Os dados foram quantificados e transpostos em planilha de banco de dados do software SPSS $15.0^{\circledR}$.

Inicialmente foi feita uma análise descritiva dos dados, relativos à distribuição por sexo, faixa etária, classificação de estado nutricional, risco de disfagia, e às médias de idade, peso e estatura.

Posteriormente, as variáveis idade, peso, estatura, risco de disfagia e estado nutricional, foram divididas em dois grupos: masculino e feminino. Testou-se estatisticamente se houve diferenças significantes entre esse dois grupos para todas as variáveis acima mencionadas. Foi verificado primeiro se as amostras, masculino e feminino, provêm de uma população com distribuição normal por meio do teste de normalidade de Shapiro-Wilks. De acordo com esse teste, as variáveis estatura e estado nutricional apresentam distribuição normal e, assim, foi aplicado o teste $t$ de Student para duas amostragens (grupos) independentes. Para as variáveis que não apresentaram normalidade, idade, peso e risco de disfagia, foi realizado o teste não-paramétrico Mann-Whitney para 2 grupos independentes. Os dois testes foram realizados com $95 \%$ de confiança $(p<0,05)$.

Entre os quatro grupos de classificação do risco de disfagia (mínimo, leve, moderado e grave), para detectar diferenças significantes entres eles para as variáveis idade, peso, estatura, risco de disfagia e estado nutricional, foi realizada a análise de variância (ANOVA), após serem feitas suposições de normalidade dos grupos e de igualdade de variâncias entre os mesmos. O resultado mostrou-se satisfatório para as variáveis: 
peso, idade e estatura, com 95\% de confiança. Para as outras variáveis, risco de disfagia e estado nutricional, que não satisfizeram o pressuposto, utilizou-se o teste não-paramétrico Kruskal-Wallis, com $95 \%$ de confiança. Por último, na análise entre o risco de disfagia e sua relação com a MAN foi feito utilizado o teste de correlação de Pearson entre essas duas variáveis.

\section{RESULTADOS}

Neste estudo foram incluídos 49 pacientes idosos, sendo 29 do sexo masculino (59\%) e 20 do feminino (41\%). A média de idade foi de 69 anos (mínimo de 60 anos e máximo de 92 anos), prevalecendo os pacientes com idade entre 60 - 74 anos e 11 meses, correspondendo a 78\% do total da amostra.

A média de peso do total da amostra foi de $59,2 \mathrm{~kg}$ (desvio-padrão - $D P=12,7 \mathrm{~kg}$ ), sendo a média do grupo masculino de 60,3 ( $D P=12,8 \mathrm{~kg}$ ) e do feminino de $57,5(D P=12,7 \mathrm{~kg})$, sem diferença significante $(p=0,846)$.

A estatura média da amostra foi de 1,62m, sendo os homens $(1,66, \mathrm{DP}=0,07 \mathrm{~m})$ mais altos que as mulheres $(1,57, \mathrm{DP}=0,07 \mathrm{~m}), \operatorname{com} p<0,0001$. Assim, há evidências para rejeitar a hipótese de que as estaturas dos dois grupos são iguais, com diferença estatisticamente significante entre eles.

A distribuição do diagnóstico nutricional, segundo a classificação da MAN, pode ser vista na Tabela 1.

Tabela 1. Distribuição de idosos internados em hospital universitário, segundo a freqüência de desnutrição. Brasília (DF) setembro, 2006.

\begin{tabular}{lll}
\hline Mini avaliação nutricional* & $\mathrm{n}$ & $\%$ \\
\hline Adequado & 14 & 29 \\
Risco de desnutrição & 25 & 51 \\
Desnutrido & 10 & 20 \\
\hline Total & 49 & 100 \\
\hline
\end{tabular}

*Classificação de acordo com a mini avaliação nutricional
Neste estudo verificou-se, no sexo masculino, uma menor diferença entre o estado nutricional adequado (34\%) e o estado nutricional inadequado (66\%), sendo $45 \%$ dos pacientes em risco nutricional e $21 \%$ desnutridos. Por outro lado, no sexo feminino essa diferença apresentou-se maior, com a freqüência, para o estado nutricional adequado, de $20 \%$ e para o estado nutricional inadequado de $80 \%$ (60\% em risco nutricional e 20\% de mulheres desnutridas).

No entanto, não há evidências pra rejeitar a hipótese de que as amostras dos grupos, masculino e feminino, são iguais $(p=0,437)$, pois não foi observada diferença significante na média do score global da MAN entre os sexos, mas ambos os sexos se encontravam em risco nutricional, masculino $(20,5, D P=5)$ e feminino $(19, D P=6)$.

Em relação ao risco de disfagia, $31 \%$ dos pacientes apresentaram risco mínimo, 47\%, leve, $18 \%$, moderado e $4 \%$, grave.

Ao comparar as médias dos valores das variáveis: estatura, peso, idade entre os quatro grupos apontados acima, foram identificadas diferenças significantes: estatura $(p=0,018)$; peso $(p=0,007)$ e idade $(p=0,034)$. O mesmo foi observado para o score do risco de disfagia $(p=0,00<0,05)$ e estado nutricional $(p<0,0001)$.

Além disso, verificou-se que, no sexo masculino, houve uma menor diferença entre o risco de disfagia mínimo (34\%) e o risco maior (66\%), sendo $41 \%$ leve, $24 \%$ moderado e $0 \%$ em risco grave. Já no sexo feminino, esta diferença apresentou-se mais elevada, com uma freqüência de risco de disfagia mínimo de $25 \%$ e $75 \%$ com risco maior, sendo $55 \%$ em risco leve, $10 \%$, moderado e $10 \%$ grave.

A análise dos quatro grupos de classificação de risco de disfagia é vista na Tabela 2 e a distribuição da classificação do risco de disfagia segundo a MAN é apresentada na Tabela 3.

Quando analisada a correlação entre os valores do risco de disfagia e seus respectivos scores da MAN, foi identificada correlação negativa moderada, de acordo com o coeficiente de correlação de Pearson (cc) de -0,61 (Figura 1). 
Tabela 2. Distribuição de idosos internados em hospital universitário (média - M e desvio-padrão - DP) por variáveis antropométricas, segundo o risco de disfagia. Brasília (DF) setembro, 2006.

\begin{tabular}{|c|c|c|c|c|c|c|c|c|c|}
\hline \multirow{3}{*}{ Variáveis } & \multicolumn{8}{|c|}{ Risco de disfagia } & \multirow{3}{*}{$p$} \\
\hline & \multicolumn{2}{|c|}{ Mínimo $(n=15)$} & \multicolumn{2}{|c|}{ Leve $(n=23)$} & \multicolumn{2}{|c|}{ Moderado $(n=9)$} & \multicolumn{2}{|c|}{ Grave $(n=2)$} & \\
\hline & $M$ & DP & $M$ & $\mathrm{DP}$ & $\mathrm{M}$ & $\mathrm{DP}$ & $\mathrm{M}$ & $\mathrm{DP}$ & \\
\hline Idade (anos) & 67 & 6 & 70 & 8 & 68 & 6 & 83 & 13 & $p<0,05$ \\
\hline Peso $(\mathrm{kg})$ & 61,6 & 8,9 & 60,4 & 12,9 & 58,5 & 11,8 & 30,0 & 2,8 & $p<0,05$ \\
\hline Estatura (m) & 1,62 & 0,06 & 1,60 & 0,08 & 1,69 & 0,09 & 1,53 & 0,03 & $p<0,05$ \\
\hline Score MAN global & 24 & 4 & 19 & 4 & 18 & 4 & 7 & 2 & $p<0,05$ \\
\hline
\end{tabular}

MAN: mini avaliação nutricional; $\mu$ : média da amostra estudada; $n$ : número de pacientes de cada grupo.

Nota: Grupo de risco de disfagia grave: como a amostra é muito pequena, não foi possível calcular o intervalo de confiança, pois não se aproxima de uma distribuição normal; Estimativa de amostra populacional, com intervalo de confiança de $95 \%$.

Tabela 3. Distribuição de pacientes idosos internados em hospital universitário, segundo risco de disfagia (conforme classificação da mini avaliação nutricional). Brasília (DF) setembro, 2006.

\begin{tabular}{|c|c|c|c|c|c|c|c|c|}
\hline \multirow{3}{*}{ Risco de disfagia } & \multicolumn{8}{|c|}{ Mini avaliação nutricional } \\
\hline & \multicolumn{2}{|c|}{ Adequado } & \multicolumn{2}{|c|}{ Risco nutricional } & \multicolumn{2}{|c|}{ Desnutrição } & \multicolumn{2}{|c|}{ Total } \\
\hline & $n$ & $\%$ & $n$ & $\%$ & $\mathrm{n}$ & $\%$ & $\mathrm{n}$ & $\%$ \\
\hline Mínimo & 10 & 67 & 5 & 33 & - & - & 15 & 100 \\
\hline Leve & 3 & 13 & 17 & 74 & 3 & 13 & 23 & 100 \\
\hline Moderado & 1 & 11 & 3 & 33 & 5 & 56 & 9 & 100 \\
\hline Grave & - & - & - & - & 2 & 100 & 2 & 100 \\
\hline Total & 14 & 29 & 25 & 51 & 10 & 20 & 49 & 100 \\
\hline
\end{tabular}

Nota: Sinal convencional utilizado; Dado numérico igual a zero, não resultante de arredondamento.



Figura 1. Correlação entre os valores do risco de disfagia e da mini avaliação nutricional em idosos internados na clínica médica do hospital universitário de Brasília. Brasília (DF), 2006.

Nota: MAN: mini avaliação nutricional; CC: coeficiente de correlação de Pearson.

Ao analisar os valores da MAN apresentados por cada grupo, conforme a classificação do risco de disfagia, utilizando o gráfico de correlação, observa-se que o grupo com risco de disfagia mínimo apresentou correlação negativa moderada $(c c=-0,536)$ entre os valores do risco de disfagia e os respectivos scores da MAN; o grupo com risco de disfagia leve não apresentou correlação $(c c=-0,098)$; risco de disfagia moderado apresentou correlação negativa moderada ( $c c=-0,528$ ) e o grupo com risco de disfagia grave apresentou correlação positiva perfeita $(\mathrm{cc}=+1,0)$.

De acordo com o sexo, ambos apresentaram correlação negativa moderada ( $c c=-0,466$ para o masculino e $\mathrm{cc}=-0,788$ para o feminino).

Dentro da classificação do risco de disfagia e de acordo com o sexo, a análise dos valores mostrou resultados diversos, sendo que foi encontrada correlação negativa perfeita entre as mulheres com risco moderado $(c c=-1,0)$; forte correlação negativa entre as mulheres com risco mínimo (cc $=-0,825)$ e correlação negativa 
moderada entre os homens com risco moderado ( $c c=-0,516)$. Houve pouca correlação entre os homens com risco mínimo ( $c c=-0,353$ ) e leve $(\mathrm{cc}=-0,171)$ e entre as mulheres com risco leve ( $c c=-0,178)$. Houve correlação positiva perfeita entre as mulheres com risco grave $(\mathrm{cc}=+1,0)$.

\section{DIS CUSS Ã O}

Um estudo prospectivo, desenvolvido por Chumlea et al..$^{29}$, mostrou um resultado semeIhante ao deste estudo em relação à maior estatura dos homens. Aquele acompanhou idosos durante seis anos, com aferições anuais de peso e estatura, e identificou que os homens são mais pesados e mais altos que as mulheres, com diferença estatística. Resultados semelhantes foram encontrados nos estudos de Perissinoto et al. ${ }^{30}$, com uma amostra randomizada de 3356 sujeitos idosos italianos, e nos de Janssen et al. ${ }^{31}$, com 468 indivíduos entre 18-88 anos.

A freqüência do estado nutricional inadequado apresentada pelos idosos, com 71,0\% em risco de desnutrição $(51,0 \%)$ ou em desnutrição $(20,0 \%)$ foi diferente do encontrado no estudo do IBRANUTRI ${ }^{3}$, realizado com pacientes adultos internados no Hospital de Base do Distrito Federal (HBDF). Esse estudo constatou que, na Região Centro-Oeste havia $27,9 \%$ de desnutridos moderados e $6,9 \%$ de desnutridos graves. A prevalência de não desnutridos foi de $65,2 \%$.

O estudo do IBRANUTRI ${ }^{3}$ envolveu quatro mil pacientes e o resultado por região identificou, na região Centro-Oeste, uma realidade diferenciada em relação às demais, visto que houve uma menor prevalência de desnutrição (34,8\%), o que poderia estar relacionado com as características da unidade hospitalar, da clínica selecionada e da amostra estudada, contemplando adultos e idosos. De modo que outro estudo similar poderia ser realizado no Distrito Federal para melhor esclarecer sobre a desnutrição hospitalar nesse local.

O estudo de Thomas et al. ${ }^{32}$, com 489 pacientes submetidos a MAN na primeira admissão hospitalar, encontrou uma prevalência mais elevada de desnutridos $(28,8 \%)$ e em risco de desnutrição $(62,5 \%)$, sendo somente $8,7 \%$ dos pacientes com estado nutricional adequado.

A tendência maior para desnutrição entre as mulheres deste estudo também foi verificada em estudo de Tavares \& Anjos $^{33}$, avaliando a Pesquisa Nacional sobre Saúde e Nutrição (PNSN), em que foram apurados os indicadores da situação nutricional da população brasileira. Seus dados mostraram que o grupo feminino apresentou maiores prevalências de formas moderadas e graves de desnutrição em quase todas as faixas etárias, com quadro de maior gravidade e alto risco para desnutrição, como visto também no estudo de Perissinoto et al..$^{30}$, em que as mulheres se encontraram desnutridas $(4,3 \%)$ em relação aos homens (2,7\%).

Isso pode ser conseqüência do fato de as mulheres serem mais afetadas pela desnutrição em período de escassez alimentar em países pobres, além do que as mulheres são predominantes entre os idosos e padecem de mais solidão, de pouca saúde e de rendimentos mais exíguos ${ }^{33}$. Apesar de o último censo de 2000 indicar uma longevidade aumentada para as mulheres, estas podem estar com a qualidade de vida prejudicada ${ }^{34,33}$.

Em relação ao risco de disfagia, a freqüência foi semelhante à identificada no estudo de Schelp et al..$^{35}$, que avaliou todos os pacientes que deram entrada em hospital universitário de referência no período de um ano, tão logo apresentassem condições para avaliação clínica, fonoaudiológica e neurológica (102 pacientes), com análise objetiva da deglutição (61 pacientes). Foi observada incidência de disfagia em 76,5\% dos pacientes avaliados clinicamente e esse percentual elevou-se a 91,0\% com avaliação videofluoroscópica.

Outros estudos apontados por Schelp et al. ${ }^{35}$ avaliaram a prevalência dos distúrbios da deglutição em pacientes internados, incluindo quadros nosológicos indiscriminados, com percentual em torno de $12 \%$. Diversos estudos ${ }^{36-38}$ que abordaram 
os aspectos epidemiológicos e a história natural da disfagia, associada a quadros vasculares encefálicos agudos, apontam para uma incidência em torno de $50 \%$ para distúrbios da deglutição. $\mathrm{Na}$ maioria destes, os pacientes foram avaliados para presença de disfagia no momento da admissão, com avaliação de deglutição clínica e videofluoroscópica, nos primeiros dias após internação.

Outro estudo, de Memg et al. ${ }^{39}$, que analisou pacientes pré-selecionados, demonstrou disfagia em $65 \%$ a $81 \%$ dos enfermos com Acidente Vascular Encefálico (AVE) localizado em tronco encefálico. $O$ estudo de Smithard et al. ${ }^{37}$ avaliou 121 pacientes com diagnóstico de AVE, tendo sido registrada disfagia, no dia da admissão hospitalar, em 61 (51\%) pacientes.

Gordon et al. ${ }^{40}$ realizaram estudo prospectivo para registrar a incidência de disfagia em 100 pacientes admitidos em período de 6 meses, em hospital geral, e determinaram a presença de disfagia em $45 \%$ dos pacientes.

Outros autores, como Mann et al. ${ }^{41}$, em análise de 128 pacientes, no período de um ano, encontraram disfagia, determinada clinicamente, em 65 (51\%) pacientes. No estudo referido, foi realizada avaliação no leito, com mediana de 3 dias.

Muitos pacientes admitidos no hospital após episódios de derrame apresentarão problema transitório ou persistente com sua deglutição. 0 resultado dessa desordem na deglutição é o prejuízo na manutenção do estado nutricional e na hidratação, ocasionando debilitação de órgãos vitais, aumento da suscetibilidade para infecções e aumento na mortalidade ${ }^{37,42}$.

Um trabalho de revisão de Martino et al. ${ }^{43}$ apontou que a incidência de disfagia, utilizando técnicas de triagem, foi baixa, variando entre 37\% e $45 \%$, aumentando após a realização de testes clínicos (51\% a 55\%) e mais ainda utilizando testes instrumentais (64\% a $78 \%$ ). Houve um aumento do risco de pneumonia em pacientes com disfagia e um grande risco em pacientes com aspiração.
O estudo transversal de Álvares-da-Silva et al. ${ }^{44}$, com 23 pacientes com Acidente Vascular Cerebral (AVC) admitidos na Sala de Observação da Emergência do Hospital de Clínicas de Porto Alegre, identificou uma proporção de $17,4 \%$ de disfagia, porém sem relação com infecção respiratória, hidratação e evolução clínica. Diante desse risco de pneumonia, compondo um quadro de infecção respiratória, a disfagia parece tornar-se um ciclo vicioso.

Os resultados deste estudo são semeIhantes aos de outros com idosos, em que, entre várias complicações decorrentes da disfagia, há a interferência no estado nutricional. No estudo de Cortés et al. ${ }^{45}$, com idosos desnutridos submetidos à avaliação fonoaudiológica específica, concluiuse que esses idosos são mais susceptíveis ao desenvolvimento de alterações de deglutição, necessitando da atuação conjunta de nutricionistas e fonoaudiólogos para que as dificuldades presentes sejam sanadas, prevenindo o aparecimento de outras alterações e garantindo a melhoria da qualidade de vida.

Estudo de Martineau et al. ${ }^{46}$ avaliou o estado nutricional, por meio da avaliação subjetiva global, de 73 pacientes admitidos em um hospital privado australiano, em fase de derrame agudo. Esse estudo detectou que, na admissão, 19,2\% dos pacientes eram desnutridos e apresentaram uma freqüência maior de disfagia $(71,0 \%)$ e de alimentação via sonda enteral (93,0\%), além de maior tempo de internação (13 dias) e outras complicações (50,0\%).

Assim, foi verificado que a freqüência de risco de disfagia dos pacientes idosos, no momento da admissão na clínica médica do HUB, é elevada $(69,0 \%)$, bem como a freqüência de estado nutricional inadequado $(71,0 \%)$.

É importante ressaltar o fato de que a avaliação nutricional realizada no momento da admissão hospitalar, com objetivo de identificar o risco de desnutrição, é o primeiro passo para o tratamento da desnutrição e a prevenção de complicações relacionadas a esse estado nutricionall$^{6}$. Assim, após essa primeira identificação, 
tornam-se mais relevantes a intervenção nutricional adequada e precoce e o acompanhamento da evolução clínica e nutricional, de modo a alcançar e avaliar os objetivos propostos.

Estudo de Sanches et al. ${ }^{47}$ avaliou idosos com demência e foi observado que a maioria desses idosos apresentou dificuldades durante a alimentação ou em atividades envolvidas nesta função, como o preparo do alimento, o manuseio de utensílios e a higiene oral, dependendo da assistência de um cuidador. Com isso, concluiuse que os idosos com demência precisam de acompanhamento fonoaudiológico e nutricional periódico, a fim de minimizar suas limitações/ incapacidades relacionadas à alimentação, prevenindo a desnutrição, a desidratação e outras complicações.

O estudo de Carnaby et al. ${ }^{48}$ comparou intervenções comportamentais de baixa e alta intensidade com cuidados usuais da disfagia, aplicados em 306 pacientes com disfagia clínica admitidos no hospital com derrame agudo. Foi observado que, comparando com pacientes em cuidados usuais e em intervenções de baixa intensidade, a terapia de intervenção de alta intensidade foi associada ao aumento da proporção de pacientes que retornaram à dieta normal e recuperaram a deglutição normal em 6 meses. Assim, esses dados mostram condutas favoráveis para pacientes em fase de derrame agudo, inseridos em programa de intervenção comportamental da deglutição, incluindo terapia ativa e modificações dietéticas ${ }^{48}$.

Ao comparar os resultados deste estudo com os de outros, percebe-se que as diferenças na identificação da freqüência de disfagia podem ser decorrentes de distintas metodologias, do período de avaliação inicial e do acompanhamento e de doença de base apresentada pelos pacientes avaliados.

Mas todos os estudos parecem apresentar um ponto em comum, reforçado com o estudo de Schelp et al. ${ }^{35}$, em que foi observada uma alta incidência de disfagia em pacientes com amplo espectro de gravidade, em diferentes fases de recuperação. Tal estudo ainda ressalta a importância de equipe multidisciplinar, incluindo fonoaudiólogos capacitados, para avaliar os distúrbios da deglutição nos diversos momentos de recuperação das AVEs.

Isso reforça o que Hudson et al. ${ }^{49}$ descrevem em seu artigo, no qual a identificação precoce do estado nutricional e a subseqüente intervenção em idosos disfágicos podem amenizar os efeitos deletérios da desnutrição.

Dessa forma, a equipe de Nutrição do serviço deve estar alerta ao risco de disfagia e ao risco nutricional, de forma a iniciar uma intervenção nutricional o mais precocemente possível; além de poder contactar o serviço de Fonoaudiologia para iniciar sua intervenção e regredir o agravamento do risco de disfagia apontado por esses pacientes. Além disso, estudos mais específicos, considerando doenças de base distintas e agregando avaliações fonoaudiológicas específicas para diagnósticos de disfagia, são necessários, de modo a estabelecer protocolos de identificação de risco mais precisos e com associação mais precisa ao estado nutricional.

\section{COLABORADORES}

J.R.V. MACIEL, autora principal, elaborou o artigo. C.J.R. OLIVEIRA, co-orientador, colaborou na revisão e orientou a elaboração do artigo. C.M.P. TADA, orientadora, colaborou na revisão, em todas as etapas do artigo, e orientou a elaboração e a finalização do artigo.

\section{REFERÊ N CIAS}

1. Organización Mundial de la Salud. Adultos de 60 o más años de edad. In: El estado físico: uso e interpretación de la antropometria. Genebra; 1995.

2. Brasília, DF. Secretaria de Estado de Saúde do Distrito Federal (SES/DF). Saúde do idoso: "A melhor idade é aquela que você tem" [acesso 2006 fev 20]. Disponível em: <http://www.saude.df. gov.br>

3. Inquérito Brasileiro de Avaliação Nutricional Hospitalar-IBRANUTRI. In: Sociedade Brasileira de 
Nutrição Parenteral e Enteral-SBPNE. Proposta para tratamento da desnutrição hospitalar no Brasil. São Paulo; 1996.

4. Mias C, Jürschik P, Massoni T, Sadurni M, Aguilà JJ, Solá R, et al. Evaluación del estado nutricional de los pacientes mayores atendidos en una unidade de hospitalización a domicilio. Nutr Hosp. 2003; 18(1):6-14

5. Guigoz Y, Lauque S, Vellas BJ. Identifying the elderly at risk for malnutrition. The mini nutritional assessment. Clin Geriatr Med. 2002; 18(4):737-57.

6. Corish CA, Kennedy NP. Protein-energy undernutrition in hospital in-patients. $\mathrm{Br} J$ Nutr. 2000; 83(6):575-91.

7. Waitzberg DL, Ferrini MT. Exame físico e antropometria. In: Waitzberg DL. Nutrição oral, enteral e parenteral na prática clínica. 3a. ed. São Paulo: Atheneu; 2000.

8. Pablo AM, Izaga MA, Alday LA. Assessment of nutritional status on hospital admission: nutritional scores. Eur J Clin Nutr. 2003; 57(7): 824-31.

9. Wyszynski DF, Perman M, Crivelli A. Prevalence of hospital malnutrition in Argentina. Preliminary results of a population-based study. Nutrition. 2003; 19(2):115-9.

10. Junqueira JCS, Soares EC, Filho HRC, Hoehr NF, Magro DO, Ueno M. Nutritional risk factors for postoperative complications in brazilian elderly patients undergoing major elective surgery. Nutrition. 2003; 19(4):312-26.

11. Ramon JM, Subira C. Prevalencia de malnutrición en la población anciana española. Med Clin (Barc.). 2001; 117(20):766-70.

12. Guigoz Y, Vellas B. A Mini avaliação nutricional (MAN) na classificação do estado nutricional do paciente idoso: apresentação, história e validação da MAN. In: Mini Avaliação Nutricional (MAN): pesquisa e prática no idoso. Nestlé Nutr Workshop Ser Clin Perform Programme. 1998; 1:01-02.

13. Guigoz Y, Vellas B, Garry PJ. Assessing the nutritional status of the elderly: the mini nutritional assessment as part of the geriatric evaluation. Nutr Rev. 1996; 54(1):S59-S65.

14. Bauduer F, Scribans C, Dubernete E, Capdupuv C. Evaluation of the nutritional status of patients over 60 -year admitted in a hematology department using the mininutritional assessment (MNA). A single centre study of 120 cases. J Nutr Health Aging. 2003; 7(3):179-82.

15. Vellas B, Guigoz Y, Baumgartner M, Garry PJ, Lauques S, Albarede JL. Relação entre marcadores nutricionais e a mini avaliação nutricional em 155 idosos. J Am Geriatric Soc. 2000; 48(10):1300-9.
16. Persson MD, Brismar KE, Katzarski KS, Nordenström J, Cederholm TE. Nutritional status using mini nutritional assessment and subjective global assessment predict mortality in geriatrics patients. J Am Geriatr Soc. 2002; 50(12):1996-2002.

17. Barone L, Milosavljevic M, Gazibarich B. Assessing the older person: is the MNA a more appropriate nutritional assessment tool than the SGA? J Nutr Health Aging. 2003; 7(1):13-7.

18. Christensson L, Unosson M, Ek AC. Evaluation of nutritional techniques in elderly people newly admitted to municipal care. Eur J Clin Nutr. 2002; 56(9):810-8.

19. Marchesan IA. Disfagia. In: Tópicos de fonoaudiologia. São Paulo: Lovise; 1995. v.2.

20. Botelho Trelis JJ, Ferrero López MI. Management of dysphagia in the institutionalized elderly patient: current situation. Nutr Hosp. 2002; 17(3):168-74.

21. Finiels H, Strudel D, Jacquot JM. Deglutition disorders in the elderly. Epidemiological aspects. Presse Med. 2001; 30(33):1623-34.

22. Macedo EDG, Furkim AM. Manual de cuidados do paciente com disfagia. São Paulo: Lovise; 2000.

23. Achem SR, Devault KR. Dysphagia in aging. J Clin Gastroenterol. 2005; 39(5):357-71.

24. Waitzberg DL, Ferrini MT. Exame físico e antropometria. In: Waitzberg DL, organizador. Nutrição oral, enteral e parenteral na prática clínica. 3a.ed. São Paulo: Atheneu; 2000. p. 255-78.

25. Chumlea WC, Roche AF, Steinbaugh ML. Estimating stature from knee height for persons 60 to 90 years of age. J Am Geriatric Soc. 1985; 33(2):116-20.

26. Sampaio HAC, Melo MLP, Almeida PC, Benevides ABP. Aplicabilidade das fórmulas de estimativa de peso e altura para idosos e adultos. Rev Bras Nutr Clin. 2002; 17(4): 117-21.

27. Guigoz Y, Vellas B, Garry PJ. Mini nutritional assessment: a practical assessment tool for grading the nutritional state of elderly patients. Facts and Research in Gerontology. 1994; (Suppl 2):15-59.

28. Souza BBA, Martins C, Campos DJ, Balsini ID, Meyer LR. Nutrição e disfagia: guia para profissionais. Curitiba: Nutroclínica; 2003.

29. Chumlea WC, Garry PJ, Hunt WC, Rhyne RL. Distributions of a serial changes in stature and weight in a healthy elderly population. Hum Biol. 1988; 60(6):917-25.

30. Perissinoto E, Pisent C, Sergi G, Grigoletto F, Enzi G. Anthropometric measurements in the elderly: age and gender differences. Br J Nutr. 2002; 87(2):177-86 
31. Janssen I, Heymsfield SB, Wang Z, Ross R. Skeletal muscle mass and distribution in 468 men and women aged 18-88 years. J Appl Physiol. 2000; 89(1):81-8.

32. Thomas DR, Zdrowski CD, Wilson MM, Conright $\mathrm{KC}$, Lewis $C$, Tariq $S$, et al. Malnutrition in subacute care. Am J Clin Nutr. 2002; 75(2):308-13.

33. Tavares EL, Anjos LA. Perfil antropométrico da população idosa brasileira: resultados da Pesquisa Nacional sobre Saúde e Nutrição. Cad Saúde Pública. 1999; 15(4):759-68.

34. Braga PMV. Evolução da expectativa de vida no Brasil [acesso 2006 set 1]. Disponível em: <http:// www. direitodoidoso.com.br/11.html>.

35. Schelp AO, Cola PC, Gatto AR, Silva RG, Carvalho LR. Incidência de disfagia orofaríngea após acidente vascular encefálico em hospital público de referência. Arq Neuropsiquiatr. 2004; 62(2-B): 504-6.

36. Smithard DG, O'Neill PA, Park C, Moris J, Wyatt R, England $\mathrm{R}$, et al. Complications and outcome after acute stroke. Stroke. 1996; 27(7):1200-4.

37. Smithard DG, O'Neill PA, England RE, Park CL, Wyatt $R$, Martin DF, et al. The natural history of dysphagia following a stroke. Dysphagia. 1997; 12(4):188-93.

38. Ekberg $\mathrm{NH}$, Hindfeldt OR, Nilsson $\mathrm{H}$, Ekberg $\mathrm{O}$, Olsson R, Hindfelt C. Dysphagia in stroke: a prospective study quantitative aspects of swallowing in dysphagic patients. Dysphagia. 1998; 13(1):32-8

39. Memg NH, Wang TG, Lien IN. Dysphagia in patients with brainstem stroke: incidence and outcome. Am J Phys Med Rehab. 2000; 79(2):170-5.

40. Gordon C, Hewer RL, Wade DT. Dysphagia in acute stroke. Br Med J (Clin Res Ed.). 1987; 295(6595): 411-4.

41. Mann G, Hankey GJ, Cameron D. Swallowing function after stroke: prognosis and prognostic factors at G months. Stroke. 1999; 30(4):744-8.
42. Watson R. Undernutrition, weight loss and feeding difficulty in elderly patients with dementia: a nursing perspective. Rev Clin Gerontol. 1997; 7(4): 317-26.

43. Martino R, Foley N, Bhogal S, Diamant N, Speechley $M$, Teasell R. Dysphagia after stroke: incidence, diagnosis, and pulmonary complications. Stroke. 2005; 36(12):2756-63.

44. Álvares-da-Silva MR, Álvares-da-Silva AU, Saafeld V, Silvério AO. Disfagia no acidente vascular cerebral agudo: um estudo transversal. GED Gastroenterol Endosc Dig. 1993; 12(3):83-7.

45. Cortés LS, Bilton TL, Suziki H, Sanches EP, Venites $J P$, Luccia GD. Análise clínica da deglutição e dificuldades de alimentação de idosos desnutridos em acompanhamento ambulatorial. Distúrb Comum. 2003; 14(2):211-35.

46. Martineau J, Bauer JD, Isenring E, Cohen S. Malnutrition determined by the patient-generated subjective global assessment is associated with poor outcomes in acute stroke patients. Clin Nutr. 2005; 24(6):1073-7.

47. Sanches EP, Bilton T, Ramos LR. Análise descritiva da alimentação de idosos com demência. Distúrb Comun. 2000; 11(2):227-49.

48. Carnaby G, Hankey GJ, Pizzi J. Behavioural intervention for dysphagia in acute stroke: a randomised controlled trial. Lancet Neurol. 2006; 5(1):31-7.

49. Hudson HM, Daubert CR, Mills RH. The interdependency of protein-energy malnutrition, aging, and dysphagia. Dysphagia. 2000; 15(1): $31-8$.

Recebido em: 8/3/2007

Versão final reapresentada em: 1/11/2007 Aprovado em: 29/2/2008 